\title{
Searching for the Radiative Decay of the Cosmic Neutrino Background with Line-Intensity Mapping
}

\author{
José Luis Bernal, ${ }^{1, *}$ Andrea Caputo $\odot^{2,3,4, \dagger}$ Francisco Villaescusa-Navarro, ${ }^{5,6}$ and Marc Kamionkowski®1 \\ ${ }^{1}$ Department of Physics and Astronomy, Johns Hopkins University, \\ 3400 North Charles Street, Baltimore, Maryland 21218, USA \\ ${ }^{2}$ School of Physics and Astronomy, Tel-Aviv University, Tel-Aviv 69978, Israel \\ ${ }^{3}$ Department of Particle Physics and Astrophysics, Weizmann Institute of Science, Rehovot 7610001, Israel \\ ${ }^{4}$ Max-Planck-Institut für Physik (Werner-Heisenberg-Institut), Föhringer Ring 6, 80805 München, Germany \\ ${ }^{5}$ Department of Astrophysical Sciences, Princeton University, Peyton Hall, Princeton, New Jersey, 08544, USA \\ ${ }^{6}$ Center for Computational Astrophysics, 162 5th Avenue, New York, New York, 10010, USA
}

(Received 6 April 2021; revised 9 June 2021; accepted 13 August 2021; published 22 September 2021)

\begin{abstract}
We study the possibility to use line-intensity mapping (LIM) to seek photons from the radiative decay of neutrinos in the cosmic neutrino background. The Standard Model prediction for the rate for these decays is extremely small, but it can be enhanced if new physics increases the neutrino electromagnetic moments. The decay photons will appear as an interloper of astrophysical spectral lines. We propose that the neutrinodecay line can be identified with anisotropies in LIM clustering and also with the voxel intensity distribution. Ongoing and future LIM experiments will have-depending on the neutrino hierarchy, transition, and experiment considered - a sensitivity to an effective electromagnetic transition moment $\sim 10^{-12}-10^{-8}\left(m_{i} c^{2} / 0.1 \mathrm{eV}\right)^{3 / 2} \mu_{B}$, where $m_{i}$ is the mass of the decaying neutrino and $\mu_{B}$ is the Bohr magneton. This will be significantly more sensitive than cosmic microwave background spectral distortions, and it will be competitive with stellar cooling studies. As a by-product, we also report an analytic form of the one-point probability distribution function for neutrino-density fluctuations, obtained from the QUIJOTE simulations using symbolic regression.
\end{abstract}

DOI: 10.1103/PhysRevLett.127.131102

Considerable efforts are underway to study the properties of neutrinos, including their masses, mixing angles, and nature (e.g., Dirac or Majorana) [1-21]. The stability of neutrinos is also of interest. An active massive neutrino $\nu_{i}$ can decay into a lighter eigenstate $\nu_{j}$ and photon, $\gamma$, $\nu_{i} \rightarrow \nu_{j}+\gamma$ with a rate determined by electromagnetic transition moments induced via loops involving gauge bosons. The Standard Model (SM) prediction for the lifetime is $\tau_{\mathrm{SM}}=7.1 \times 10^{43} m_{\mathrm{eV}}^{-5} \mathrm{~s}[22-25]$, where $m_{\mathrm{eV}} \equiv$ $m_{\nu} c^{2} / \mathrm{eV}$ is the neutrino mass in $\mathrm{eV} / c^{2}$ units, significantly longer than the age of the Universe.

However, new physics beyond the SM (BSM) can enhance neutrino magnetic moments [26-34], and such modifications have been considered in connection with experimental anomalies, such as a possible correlation of solar neutrinos with solar activity [14,35], or more recently [33,34] the $\sim 3 \sigma$ excess reported XENON1T [36]. Although many avenues have been proposed (see,

Published by the American Physical Society under the terms of the Creative Commons Attribution 4.0 International license. Further distribution of this work must maintain attribution to the author(s) and the published article's title, journal citation, and DOI. Funded by SCOAP ${ }^{3}$. e.g., Ref. [37] for a review), the most efficient direct laboratory probe of neutrino electromagnetic couplings involves neutrino-electron scattering [7,8,38]. Tighter bounds on neutrino electromagnetic moments come from astrophysics. In particular, the strongest constraint comes from the tip of the red giant branch in globular clusters, which is sensitive to the additional energy loss through plasmon decay into two neutrinos [39-41]. Radiative neutrino decays have also been constrained from measurements of the cosmic microwave background (CMB) spectral distortions $[42,43]$.

Here we study the use of line-intensity mapping (LIM) to seek photons from radiative decays of neutrinos in the cosmic neutrino background. LIM [44,45] exploits the integrated intensity at a given frequency induced by a wellidentified spectral line to map the three-dimensional distribution of matter in the Universe. Photons from particle decays will appear in these maps as an unidentified line [46] that can be distinguished from astrophysical lines through its clustering anisotropies and through the voxel probability distribution function [47]. We find that LIM has the potential to be significantly more sensitive to radiative decays than current cosmological probes and compete with the strongest bounds to electromagnetic moments coming from astrophysical observations. 
While neutrino radiative decays are characterized by the electromagnetic transition moments, LIM experiments are sensitive to the luminosity density $\rho_{L}$ of the photons produced in each point $\boldsymbol{x}$, which, for the decay between the $i$ and $j$ states, is given by

$$
\rho_{L}^{i j}(\boldsymbol{x})=(1 / 6) \rho_{\nu}(\boldsymbol{x}) c^{2} \Gamma_{i j}\left(1-m_{j}^{2} / m_{i}^{2}\right),
$$

where $\rho_{\nu}$ is the total neutrino density, $\Gamma_{i j} \equiv \tau_{i j}^{-1}$ is the decay rate, and $m_{i}$ are the neutrino masses. We assume that the density of each state is $1 / 3$ of the total density, as expected apart from small mass differences and flavor corrections that have negligible consequences for the precision goals of this Letter [48]. The corresponding brightness temperature $T$ at redshift $z$ is

$$
\begin{aligned}
T^{i j}(z, \boldsymbol{x}) & =\frac{c^{3}(1+z)^{2} \rho_{L}^{i j}(z, \boldsymbol{x})}{8 \pi k_{B} f^{3} H(z)}=X_{L T} \rho_{L}^{i j}(z, \boldsymbol{x}) \\
& =\left(X_{L T} / 6\right) \rho_{\nu} c^{2} \Gamma_{i j}\left(1-m_{j}^{2} / m_{i}^{2}\right),
\end{aligned}
$$

where $H$ is the Hubble expansion and $k_{B}$ is the Boltzmann constant and $f$ is the rest-frame frequency [49]. Thus, the brightness temperature from neutrino decays traces the neutrino-density field.

Decay photons are then an emission line with rest-frame frequency given by $f_{i j}=\left(m_{i}^{2}-m_{j}^{2}\right) c^{2} /\left(2 h_{P} m_{i}\right)$, where $h_{P}$ is the Planck constant. For $m_{i} / c^{2} \gg T_{\nu} / k_{B} \sim 10^{-4}(1+$ $z) \mathrm{eV}$ (where $T_{\nu}$ is the cosmic neutrino temperature), which holds true for our cases of interest, the neutrinos are nonrelativistic, and we can neglect the linewidths due to their velocity dispersion [50]. The rest-frame frequency of the emission lines is then uniquely characterized by the neutrino hierarchy and the sum $\sum m_{\nu}$ of neutrino masses, as shown in Fig. 1, with the observed frequency redshifted accordingly. The transitions not included in the figure have a frequency very similar to one of the other two (e.g., $f_{31} \approx$ $f_{32}$ for the normal hierarchy) and are not distinguished hereinafter.

We now consider two LIM observables: the power spectrum and the voxel intensity distribution (VID). The observed anisotropic LIM power spectrum associated to the neutrino decay between $i$ and $j$ states is [47,51]

$$
P_{i j}(k, \mu)=W(k, \mu) X_{L T}^{2}\left\langle\rho_{L}^{i j}\right\rangle^{2} F_{\mathrm{RSD}}^{2}(k, \mu) P_{\nu}(k),
$$

where $k$ is the modulus of the Fourier mode, $\mu \equiv \boldsymbol{k} \cdot \boldsymbol{k}_{\|} / k^{2}$ is the cosine of the angle between the Fourier mode and the line of sight, $W$ is a window function modeling the effects from instrumental resolution and finite volume observed, the brackets \langle\rangle denote the spatial mean, $F_{\mathrm{RSD}}$ is a redshift-space distortions factor [51], $P_{\nu}$ is the neutrino power spectrum, computed using CAMB [52], and all redshift dependence is implicit. We consider the
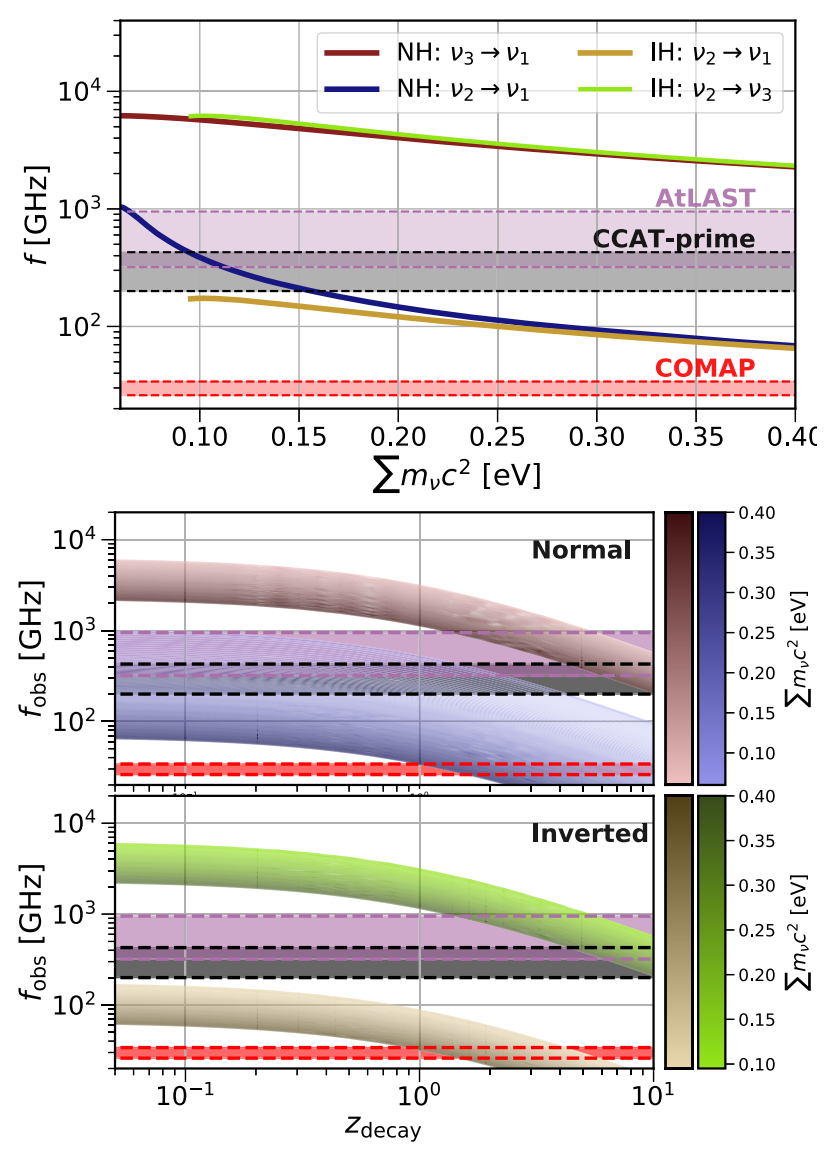

FIG. 1. Relation between the sum of neutrino masses and the rest-frame frequency of the photon produced in the decay for $\mathrm{NH}$ and $\mathrm{IH}$ (top) and the corresponding observed frequency. We also show the frequency bands of the experiments considered in both panels with horizontal shaded bands.

Legendre multipoles of the LIM power spectrum with respect to $\mu$ up to the hexadecapole.

Similarly, the VID is related to the probability distribution function (PDF) $\mathcal{P}_{\breve{\rho}}$ of the normalized total neutrino density $\breve{\rho}_{\nu} \equiv \rho_{\nu} /\left\langle\rho_{\nu}\right\rangle$, as $\mathcal{P}_{i j}(T)=\mathcal{P}_{\breve{\rho}}\left(\breve{\rho}_{\nu}\right) /\left\langle T^{i j}\right\rangle$. We estimate the neutrino-density PDF from high-resolution simulations of the QUIJOTE simulation suite [53], that model the gravitational evolution of more than 2 billion cold dark matter and neutrino particles in a comoving box of $\left(1 h^{-1} \mathrm{Gpc}\right)^{3}$ volume. Degenerate neutrino mass eigenstates are assumed.

First, neutrino particle positions are assigned to a regular grid with $1500^{3}$ voxels employing the cloud-in-cell massassignment scheme. Next, the 3D field is convolved with a Gaussian kernel of a given width. Then, the PDF is estimated by computing the fraction of voxels with a given $\breve{\rho}_{\nu}$. We do this for $\sum m_{\nu} c^{2}=\{0.1,0.2,0.4\} \mathrm{eV}$, at $z=\{0,0.5,1,2,3,4,5,6,7,8,9\}$ and for six smoothing scales $\{2,3,4,5,7.5,10\} h^{-1}$ Mpc. We have checked that the computed PDFs, in the range of interest for this study, are converged in our simulations. Note that all dependences 
can be condensed in the root-mean square $\sigma$ of smoothed density field, which depends on $\sum m_{\nu}, z$, and the smoothing scale. Finally, we use symbolic regression to approximate this grid of PDFs using the Eureqa package (https:// www.datarobot.com/nutonian/) finding

$$
\begin{aligned}
\frac{\mathcal{P}_{\breve{\rho}}}{\mathcal{A}}= & \exp \left\{\frac{0.2 \mathcal{G}\left(0.6 \frac{\mathfrak{d}}{\mathfrak{g}}\right)+2.5 \mathfrak{g}^{1.6} \mathcal{G}\left(1.1+\frac{\mathfrak{d}}{\mathfrak{g}}-2.3 \mathfrak{I}\right)}{\mathfrak{S}+0.05 \mathcal{G}\left(0.6 \frac{\mathfrak{d}}{\mathfrak{g}}\right)}\right. \\
& \left.-2.5 \mathfrak{I}^{1.6} \mathcal{G}(1.1+(\mathfrak{d} / \mathfrak{g})-2.3 \mathfrak{I})\right\}-1,
\end{aligned}
$$

where $\mathcal{G}(x) \equiv e^{-x^{2}}, \mathfrak{d} \equiv \log \breve{\rho}_{\nu}, \mathfrak{g} \equiv \log (1+\sigma)$, and $\mathcal{A}$ is a normalization factor.

LIM experiments will not target the emission line from neutrino decays, but known astrophysical lines. In turn, the neutrino-decay line will redshift into the telescope frequency band from a different redshift. All emission lines other than the main target that contribute to the total signal tracing other cosmic volumes are known as line interlopers. These contributions, if known, can be identified and modeled (see, e.g., [54-62]). However, the neutrino-decay line will be an unknown line interloper. From Fig. 1 we can see that the frequencies of interest lie in the frequency bands of experiments like COMAP [63] (which targets the CO line) and Cerro Chajnantor Atacama Telescope (CCAT)-prime [64] and Atacama Large Aperture Submillimeter Telescope (AtLAST) [65] (which target the CII line); their instrumental specifications are summarized in Table I.

We assume the fiducial astrophysical model for the $\mathrm{CO}$ and the CII lines from Refs. [66] and [67], and model their power spectrum and VID, with their corresponding covariances, following Refs. [47,51,68]. For the VID analysis, we use a modified Schechter function with the parameters reported in Ref. [47]. We take lambda cold dark matter cosmology with best-fit parameter values from Planck temperature, polarization, and lensing power spectra [69] assuming $\sum m_{\nu} c^{2}=0.06$ as our fiducial model. We consider normal $(\mathrm{NH})$ and inverted $(\mathrm{IH})$ neutrino hierarchies [70].

Recently, a similar situation, regarding decaying dark matter, was described in Ref. [47], where strategies to detect such decays were proposed. Here we adapt that modeling to the neutrino-decay case, considering neutrino decays happening at $z<10$, and perform a Fisher-matrix analysis [71-74], accounting for the uncertainty in the astrophysical model. In summary, the contribution from neutrino decays to the VID can be modeled by convoluting $\mathcal{P}_{i j}(T)$ with the astrophysical and noise VIDs: the total VID is the result of the sum of the three contributions. In turn, the contribution to the power spectrum consists of the addition of the projected power spectrum from neutrino decays to a different redshift, which introduces a strong anisotropy in the power spectrum, altering the ratio between the Legendre multipoles. For the power spectrum, we do not consider decays from the same cosmic volumes probed by the astrophysical line because they are very degenerate with astrophysical uncertainties.

We show the forecasted minimum values of $\Gamma_{i j}$ which LIM experiments will be sensitive to at the $95 \%$ confidence level, as a function of the neutrino hierarchy, transition, and $\sum m_{\nu}$ in Fig. 2. We limit the minimum $\sum m_{\nu}$ at the minimum mass allowed for each hierarchy from neutrino oscillation experiments [75]. As expected from Fig. 1, COMAP and the experiments targeting CII are sensitive to the transitions between close and far mass eigenstates, respectively (with the exception of low $\sum m_{\nu}$ in the normal hierarchy).

After marginalizing over the astrophysical uncertainties of the target line as in Ref. [47], we find that for all cases considered, LIM experiments can improve current cosmological bounds on the neutrino-decay rate from $\mathrm{CMB}$ spectral distortions $[42,43]$ by several orders of magnitude. This shows that LIM has the potential to provide the strongest cosmological sensitivity on neutrino radiative decays. Furthermore, LIM will be competitive to the most stringent limits to date, coming from stellar cooling [39-41], as we see below.

As mentioned above, at the microscopic level radiative neutrino decays may result from an effective term in the Lagrangian like $\alpha \bar{\nu}^{i} \sigma_{\alpha \beta}\left(\mu_{i j}+\epsilon_{i j} \gamma_{5}\right) \nu^{j} F^{\alpha \beta}+$ Hermitian conjugates $[14,37,76]$, where $F^{\alpha \beta}$ is the electromagnetic field tensor, $\sigma_{\alpha \beta}$ is the Dirac gamma matrices commutator, and $\mu_{i j}$ and $\epsilon_{i j}$ are the magnetic and electric moments,

TABLE I. Instrumental specifications used. Each independent frequency band is separated by commas. CII observations use the convention for specific intensity instead of brightness temperature. We combine $30 \times 30$ pixels for VID analyses with ATLAST. More

\begin{tabular}{|c|c|c|c|}
\hline Experiment & COMAP 1 (2) & CCAT-prime & ATLAST \\
\hline Line & $\mathrm{CO}$ & $\mathrm{CII}$ & $\mathrm{CII}$ \\
\hline Freq. band $[\mathrm{GHz}]$ & $24-36$ & $200-240,260-300,330-370,388-428$ & $315-376,376-470,470-620,620-920$ \\
\hline Spectral resolution & 4000 & 100 & 1000 \\
\hline Ang. resolution ["] & 240 & $57,45,35,30$ & $4.4,3.6,2.8,2.0$ \\
\hline Sky coverage $\left[\mathrm{deg}^{2}\right]$ & $2.25(60)$ & 8 & 7500 \\
\hline Voxel noise & $39(69) \mu \mathrm{K}$ & $(0.6,1.0,2.5,5.7) \times 10^{4} \mathrm{Jy} / \mathrm{sr}$ & $(0.4,0.7,1.4,3.9) \times 10^{5} \mathrm{Jy} / \mathrm{sr}$ \\
\hline
\end{tabular}
details can be found in Ref. [47]. 


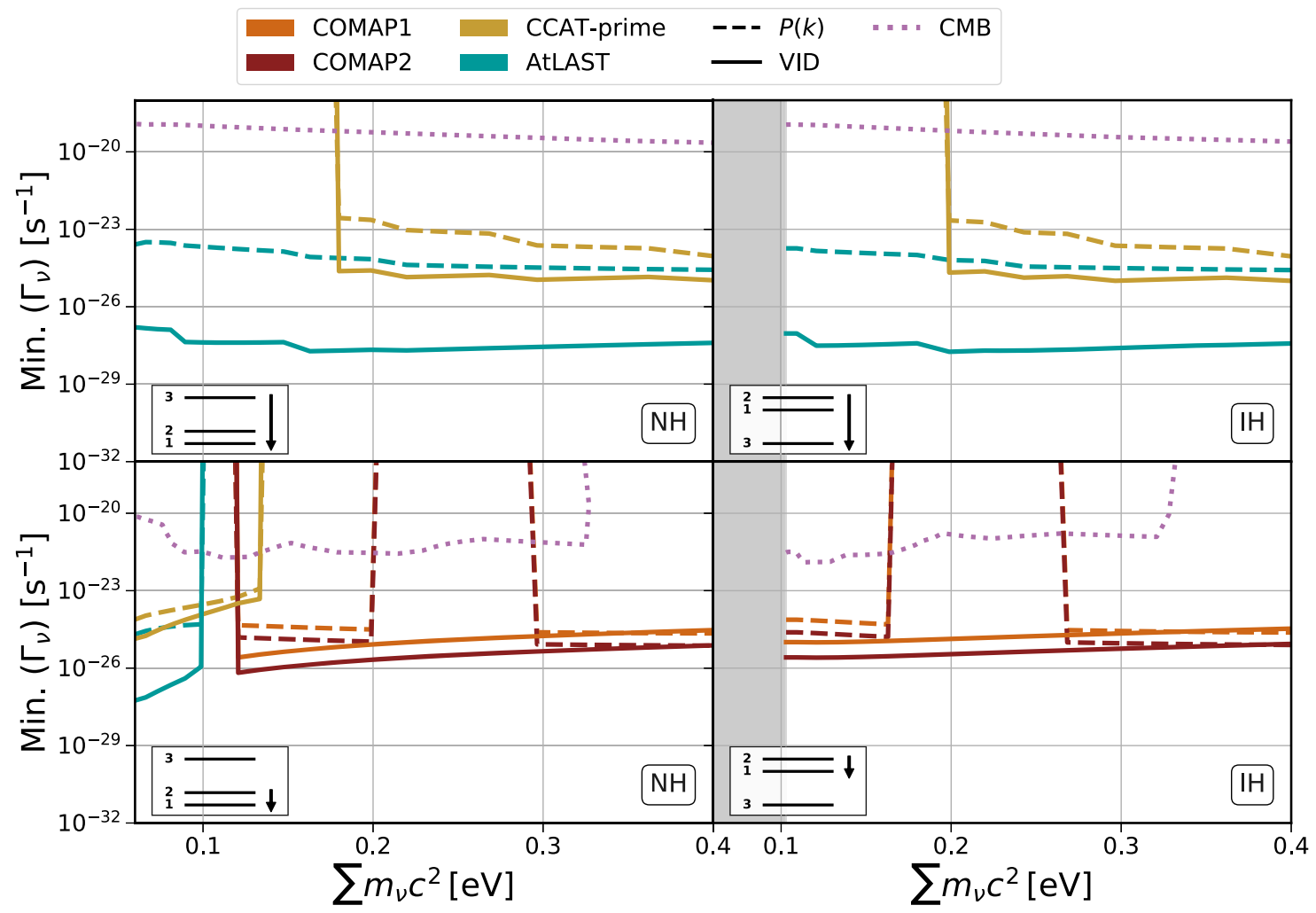

FIG. 2. Forecasted $95 \%$ confidence level marginalized upper limits of $\Theta_{\nu}$ as a function of the total neutrino mass from measurements for the power spectrum (dashed lines) and VID (solid lines) for all LIM surveys considered, namely, COMAP1 (orange), COMAP2 (red), CCAT-prime (dark yellow), and ATLAST (light blue). The left panels refer to the NH case, while the right ones to the IH case. In both cases we also indicate the considered transition between eigenstates. The dotted purple lines indicate the CMB limits from Ref. $[42,43]$.

respectively. For a transition (i.e., $i \neq j$ ), we can relate an effective electromagnetic moment $\mu_{i j}^{\text {eff }}$ to the decay rate as

$$
\left(\mu_{i j}^{\mathrm{eff}}\right)^{2} \simeq \frac{\Gamma_{i j}}{5 \mathrm{~s}^{-1}} \frac{m_{\mathrm{eV}, i}^{3}}{\left(m_{\mathrm{eV}, i}^{2}-m_{\mathrm{eV}, j}^{2}\right)^{3}} \mu_{B}^{2}
$$

where $\left|\mu_{i j}^{\text {eff }}\right|^{2} \equiv\left|\mu_{i j}\right|^{2}+\left|\epsilon_{i j}\right|^{2}$, and $\mu_{B}$ is the Bohr magneton.

According to Eq. (5), the forecasted LIM sensitivity of $\Gamma_{i j} \sim 10^{-28}-10^{-25} \mathrm{~s}^{-1}$ at $95 \%$ confidence level translates to $\mu_{i j}^{\text {eff }} \sim 10^{-12}-10^{-8}\left(m_{i} c^{2} / 0.1 \mathrm{eV}\right)^{3 / 2} \mu_{B}$, while current and forecasted $\mathrm{CMB}$ limits are $\sim 10^{-7}-10^{-8} \mu_{B}$ and $\sim 10^{-8}-3 \times 10^{-11} \mu_{B}$, respectively [43]. Note that the sensitivity to $\mu_{i j}^{\text {eff }}$ depends on the mass of the original neutrino, which in turn depends on the transition, hierarchy, and $\sum m_{\nu}$ considered. In turn, the most stringent direct detection limit was obtained in the Borexino experiment and is related to an effective moment accounting for all magnetic direct and transition moments: $\mu_{\nu}^{\text {eff }}<2.8 \times$ $10^{-11} \mu_{B}$ at $90 \%$ confidence level [77]. Finally, astrophysical studies of stellar cooling set the strongest bounds to date: $\mu_{\nu}^{\text {eff }}<4.5 \times 10^{-12} \mu_{B}$ at $95 \%$ confidence level [41].
This demonstrates the great potential that LIM surveys have to unveil neutrino properties: on top of having a sensitivity competitive to and in some cases even improving current strongest limits, LIM experiments may probe neutrino decays in a very different context than the rest of the experiments and observations discussed above. Instead of neutrinos produced in the interior of stars, LIM will be sensitive to the cosmic neutrino background (as CMB studies are, but at very different redshifts). Moreover, the energy of the neutrinos involved in each probe also varies, which may inform about a potential energy dependence of the electromagnetic transition moments [78]. These synergies are very timely, since an enhanced magnetic moment may explain the $\sim 3 \sigma$ excess observed by XENON1T [36], but the values required are close to the limits found by Borexino and in tension with stellar cooling constraints.

Finally, LIM may provide additional information about the cosmic neutrino background beyond the effect of $\sum m_{\nu}$ in the growth of perturbations: combining the information about $\sum m_{\nu}$ with the frequency of the photons produced in the decay, LIM might be the only cosmological probe sensitive to individual neutrino masses and their hierarchy [79]. 
The complementarity between different probes of neutrino decays will also help as a cross-check for eventual caveats or systematic uncertainties in the measurements. In the case of LIM experiments, these are the same as for the search for radiative dark matter decays, which are discussed in Ref. [47]. In summary, astrophysical uncertainties are already accounted for in our analysis, there are efficient strategies to deal with known astrophysical line interlopers [54-62], and galactic foregrounds are expected to be under control at the frequencies of interest. Moreover, the neutrino-decay contribution to the LIM power spectrum and VID is very characteristic, and the combination of both summary statistics will not only improve the sensitivity but also the robustness of the measurement [80]. Finally, we have assumed that the neutrino-decay line is a delta function, and neglected any widening due to the neutrino velocity distributions. While this is a good approximation for the regime of interest at this stage, it is also possible to model the neutrino-decay emissivity with a generic momentum distribution [43]; this will allow us to adapt our analysis to neutrino production models that alter their momentum distribution [82].

The neutrino-decay contribution might be confused with other exotic radiation injections such as dark matter decay. However, the shape of the neutrino power spectrum and density PDF is different. Moreover, while the contribution from dark matter decays will appear in LIM cross-correlations with galaxy clustering [46] and lensing [83], the contribution from neutrino decays will barely do so, since galaxy surveys do not trace the neutrino-density field.

In this Letter we have proposed the use of LIM for the detection of a possible radiative decay of the cosmic neutrino background, focusing on its contribution to the LIM power spectrum and VID. We have also provided a first parametric fit of the neutrino-density PDF using $\mathrm{N}$-body simulations and symbolic regression, which was required to compute the contribution to the VID. Our results show that LIM have the potential to achieve sensitivities competitive with current limits, improving other cosmological probes by several orders of magnitude. The complementarity of LIM and other existing probes of neutrino decays opens exciting synergies, as well as checks for systematics, that will lead the way to new studies of neutrino properties.

J. L. B. is supported by the Allan C. and Dorothy H. Davis Fellowship. A. C. acknowledges support from the Israel Science Foundation (Grant No. 1302/19), the USIsraeli BSF (Grant No. 2018236), and the German Israeli GIF (Grant No. I-2524-303.7). A. C. acknowledges the hospitality of the Max Planck Institute of Physics in Munich. F. V.N. acknowledges funding from the Wide Field InfraRed Survey program through NNG26PJ30C and NNN12AA01C. This work was supported at Johns Hopkins by NSF Grant No. 1818899 and the Simons Foundation. *jbernal2@jhu.edu †andrea.caputo@uv.es

[1] V. Cung and M. Yoshimura, Electromagnetic interaction of neutrinos in gauge theories of weak interactions, Riv. Nuovo Cimento Soc. Ital. Fis. 29, 557 (1975).

[2] L. Rosenberg, Electromagnetic interactions of neutrinos, Phys. Rev. 129, 2786 (1963).

[3] S. Mertens et al. (KATRIN Collaboration), A novel detector system for KATRIN to search for $\mathrm{keV}$-scale sterile neutrinos, J. Phys. G 46, 065203 (2019).

[4] A. Gando et al. (KamLAND-Zen Collaboration), Search for Majorana Neutrinos Near the Inverted Mass Hierarchy Region with KamLAND-Zen, Phys. Rev. Lett. 117, 0825031 (2016); Phys. Rev. Lett. 117, A109903 (2016).

[5] J. B. Albert et al. (EXO-200 Collaboration), Search for Majorana neutrinos with the first two years of EXO-200 data, Nature (London) 510, 229 (2014).

[6] T. Brunner and L. Winslow, Searching for $0 \nu \beta \beta$ decay in ${ }^{136} \mathrm{Xe}$ - Towards the tonne-scale and beyond, Nucl. Phys. News 27, 14 (2017).

[7] A. Beda, E. Demidova, A. Starostin, V. Brudanin, V. Egorov, D. Medvedev, M. V. Shirchenko, and A. S. Starostin, GEMMA experiment: Three years of the search for the neutrino magnetic moment, Phys. Part. Nucl. Lett. 7, 406 (2010).

[8] M. Deniz et al. (TEXONO Collaboration), Measurement of $\mathrm{Nu}(\mathrm{e})$-bar -Electron Scattering Cross-Section with a CsI(Tl) Scintillating Crystal Array at the Kuo-Sheng Nuclear Power Reactor, Phys. Rev. D 81, 072001 (2010).

[9] K. Abe et al. (T2K Collaboration), Measurement of neutrino and antineutrino oscillations by the $\mathrm{T} 2 \mathrm{~K}$ experiment including a new additional sample of $\nu_{e}$ interactions at the far detector, Phys. Rev. D 96, 092006 (2017); Phys. Rev. D 98, 019902(E) (2018).

[10] P. Adamson et al. (NOvA Collaboration), Constraints on Oscillation Parameters from $\nu_{e}$ Appearance and $\nu_{\mu}$ Disappearance in NOvA, Phys. Rev. Lett. 118, 231801 (2017).

[11] R. Acciarri et al. (DUNE Collaboration), Long-Baseline Neutrino Facility (LBNF) and Deep Underground Neutrino Experiment (DUNE): Conceptual Design Report, Volume 1: The LBNF and DUNE Projects, arXiv:1601.05471.

[12] K. Abe et al. (Hyper-Kamiokande Proto- Collaboration), Physics potential of a long-baseline neutrino oscillation experiment using a J-PARC neutrino beam and HyperKamiokande, Prog. Theor. Exp. Phys. 2015, 053C02 (2015).

[13] M. G. Aartsen et al. (IceCube Collaboration), Search for sterile neutrino mixing using three years of IceCube DeepCore data, Phys. Rev. D 95, 112002 (2017).

[14] G. Raffelt, Stars as laboratories for fundamental physics: The astrophysics of neutrinos, axions, and other weakly interacting particles, 1996.

[15] B. Pontecorvo, Neutrino experiments and the problem of conservation of leptonic charge, Sov. Phys. JETP 26, 984 (1968).

[16] V. Gribov and B. Pontecorvo, Neutrino astronomy and lepton charge, Phys. Lett. 28B, 493 (1969).

[17] P. Langacker, S. Petcov, G. Steigman, and S. Toshev, On the Mikheev-Smirnov-Wolfenstein (MSW) mechanism of 
amplification of neutrino oscillations in matter, Nucl. Phys. B282, 589 (1987).

[18] S. M. Bilenky, J. Hosek, and S. Petcov, On oscillations of neutrinos with dirac and Majorana masses, Phys. Lett. 94B, 495 (1980).

[19] E. K. Akhmedov, Neutrino physics, in Proceeding in Contribution to: ICTP Summer School in Particle Physics (1999), Vol. 6, pp. 103-164. arXiv:hep-ph/0001264.

[20] M. C. Gonzalez-Garcia and Y. Nir, Neutrino masses and mixing: Evidence and implications, Rev. Mod. Phys. 75, 345 (2003).

[21] M. C. Gonzalez-Garcia and M. Maltoni, Phenomenology with massive neutrinos, Phys. Rep. 460, 1 (2008).

[22] F. Boehm and P. Vogel, Physics of Massive Neutrinos (Cambridge University Press, 1992), https://doi.org/10 .1017/CBO9780511622571.

[23] P. B. Pal and L. Wolfenstein, Radiative decays of massive neutrinos, Phys. Rev. D 25, 766 (1982).

[24] S. T. Petcov, The Processes $\mu \rightarrow e+\gamma, \mu \rightarrow e+\bar{e}, \nu^{\prime} \rightarrow$ $\nu+\gamma$ in the Weinberg-Salam Model with Neutrino Mixing, Sov. J. Nucl. Phys. 25, 340 (1977); Yad. Fiz. 25, 1336(E) (1977) [Sov. J. Nucl. Phys. 25, 698(E) (1977).

[25] K. Fujikawa and R. Shrock, The Magnetic Moment of a Massive Neutrino and Neutrino Spin Rotation, Phys. Rev. Lett. 45, 963 (1980).

[26] N. F. Bell, M. Gorchtein, M. J. Ramsey-Musolf, P. Vogel, and P. Wang, Model independent bounds on magnetic moments of Majorana neutrinos, Phys. Lett. B 642, 377 (2006).

[27] S. Davidson, M. Gorbahn, and A. Santamaria, From transition magnetic moments to majorana neutrino masses, Phys. Lett. B 626, 151 (2005).

[28] H. Georgi and L. Randall, Charge conjugation and neutrino magnetic moments, Phys. Lett. B 244, 196 (1990).

[29] B. W. Lee and R.E. Shrock, Natural Suppression of symmetry violation in gauge theories: Muon-lepton and electron lepton number nonconservation, Phys. Rev. D 16, 1444 (1977).

[30] R. Shrock, Decay 10->nu(lepton) gamma in gauge theories of weak and electromagnetic interactions, Phys. Rev. D 9, 743 (1974).

[31] M. Lindner, B. Radovčić, and J. Welter, Revisiting large neutrino magnetic moments, J. High Energy Phys. 07 (2017) 139.

[32] M. Lindner, B. Radovčić, and J. Welter, Revisiting large neutrino magnetic moments, J. High Energy Phys. 07 (2017) 139.

[33] O. G. Miranda, D. K. Papoulias, M. Tórtola, and J. W. F. Valle, XENON1T signal from transition neutrino magnetic moments, Phys. Lett. B 808, 135685 (2020).

[34] K. S. Babu, S. Jana, and M. Lindner, Large neutrino magnetic moments in the light of recent experiments, J. High Energy Phys. 10 (2020) 040.

[35] J. N. Bahcall, Neutrino Astrophysics (Cambridge University Press, Cambridge, England, 1989).

[36] E. Aprile et al. (XENON Collaboration), Excess electronic recoil events in XENON1T, Phys. Rev. D 102, 072004 (2020).
[37] C. Giunti and A. Studenikin, Neutrino electromagnetic interactions: A window to new physics, Rev. Mod. Phys. 87, 531 (2015).

[38] P. Vogel and J. Engel, Neutrino electromagnetic form factors, Phys. Rev. D 39, 3378 (1989).

[39] G. G. Raffelt, New Bound on Neutrino Dipole Moments from Globular-Cluster Stars, Phys. Rev. Lett. 64, 2856 (1990).

[40] G. Raffelt and A. Weiss, Non-standard neutrino interactions and the evolution of red giants, Astron. Astrophys. 264, 536 (1992).

[41] N. Viaux, M. Catelan, P. B. Stetson, G. Raffelt, J. Redondo, A. A. R. Valcarce, and A. Weiss, Particle-physics constraints from the globular cluster M5: Neutrino dipole moments, Astron. Astrophys. 558, A12 (2013).

[42] A. Mirizzi, D. Montanino, and P. D. Serpico, Revisiting cosmological bounds on radiative neutrino lifetime, Phys. Rev. D 76, 053007 (2007).

[43] J. L. Aalberts et al., Precision constraints on radiative neutrino decay with $\mathrm{CMB}$ spectral distortion, Phys. Rev. D 98, 023001 (2018).

[44] E. D. Kovetz et al., Line-intensity mapping: 2017 Status Report, arXiv:1709.09066.

[45] E. D. Kovetz et al., Astrophysics and cosmology with lineintensity mapping, arXiv:1903.04496.

[46] C. Creque-Sarbinowski and M. Kamionkowski, Searching for decaying and annihilating dark matter with line intensity mapping, Phys. Rev. D 98, 063524 (2018).

[47] J. L. Bernal, A. Caputo, and M. Kamionkowski, Strategies to detect dark-matter decays with line-intensity mapping, arXiv:2012.00771.

[48] G. Mangano, G. Miele, S. Pastor, T. Pinto, O. Pisanti, and P. D. Serpico, Effects of non-standard neutrino-electron interactions on relic neutrino decoupling, Nucl. Phys. B756, 100 (2006).

[49] A. Lidz, S. R. Furlanetto, S. P. Oh, J. Aguirre, T.-C. Chang, O. Doré, and J. R. Pritchard, Intensity mapping with carbon monoxide emission lines and the redshifted $21 \mathrm{~cm}$ line, Astrophys. J. 741, 70 (2011).

[50] The widening would only be relevant if larger than the instrumental spectral resolution $f_{\text {obs }} / \delta f$, where $f_{\text {obs }}$ is the observed frequency and $\delta f$ is the channel width. In such a case, we would take the linewidth as the spectral resolution for the neutrino-decay line.

[51] J. L. Bernal, P. C. Breysse, H. Gil-Marín, and E. D. Kovetz, User's guide to extracting cosmological information from line-intensity maps, Phys. Rev. D 100, 123522 (2019).

[52] A. Lewis, A. Challinor, and A. Lasenby, Efficient computation of CMB anisotropies in closed FRW models, Astrophys. J. 538, 473 (2000).

[53] F. Villaescusa-Navarro et al., The Quijote simulations, Astrophys. J. Suppl. Ser. 250, 2 (2020).

[54] S. P. Oh and K. J. Mack, Foregrounds for 21-cm observations of neutral gas at high redshift, Mon. Not. R. Astron. Soc. 346, 456 (2003).

[55] X. Wang, M. Tegmark, M. G. Santos, and L. Knox, $21 \mathrm{~cm}$ tomography with foregrounds, Astrophys. J. 650, 529 (2006). 
[56] A. Liu and M. Tegmark, A method for $21 \mathrm{~cm}$ power spectrum estimation in the presence of foregrounds, Phys. Rev. D 83, 103006 (2011).

[57] P. C. Breysse, E. D. Kovetz, and M. Kamionkowski, Masking line foregrounds in intensity-mapping surveys, Mon. Not. R. Astron. Soc. 452, 3408 (2015).

[58] A. Lidz and J. Taylor, On removing interloper contamination from intensity mapping power spectrum measurements, Astrophys. J. 825, 143 (2016).

[59] G. Sun, L. Moncelsi, M. P. Viero, M. B. Silva, J. Bock, C. M. Bradford et al., A foreground masking strategy for [C II] intensity mapping experiments using galaxies selected by stellar mass and redshift, Astrophys. J. 856, 107 (2018).

[60] Y.-T. Cheng, T.-C. Chang, J. Bock, C. M. Bradford, and A. Cooray, Spectral line de-confusion in an intensity mapping survey, Astrophys. J. 832, 165 (2016).

[61] Y.-T. Cheng, T.-C. Chang, and J. J. Bock, Phase-space spectral line deconfusion in intensity mapping, Astrophys. J. 901, 142 (2020).

[62] Y. Gong, X. Chen, and A. Cooray, Cosmological Constraints from Line Intensity Mapping with Interlopers, Astrophys. J. 894, 152 (2020).

[63] K. Cleary, M.-A. Bigot-Sazy, D. Chung et al., The CO mapping array pathfinder (COMAP), AAS Meeting Abstracts 227, 426 (2016).

[64] S. K. Choi et al., Sensitivity of the Prime-Cam Instrument on the CCAT-Prime Telescope, J. Low Temp. Phys. 199, 1089 (2020).

[65] P. D. Klaassen, T. K. Mroczkowski, C. Cicone, E. Hatziminaoglou, S. Sartori, C. De Breuck et al., The Atacama Large Aperture Submillimeter Telescope (AtLAST), in Society of Photo-Optical Instrumentation Engineers (SPIE) Conference Series, Vol. 11445 of Society of Photo-Optical Instrumentation Engineers (SPIE) Conference Series (2020), p. 114452F, https://dx.doi.org/ $10.1117 / 12.2561315$.

[66] T. Y. Li, R. H. Wechsler, K. Devaraj, and S. E. Church, Connecting CO intensity mapping to molecular gas and star formation in the epoch of galaxy assembly, Astrophys. J. 817, 169 (2016).

[67] M. Silva, M. G. Santos, A. Cooray, and Y. Gong, Prospects for Detecting C II Emission during the Epoch of Reionization, Astrophys. J. 806, 209 (2015).

[68] P. C. Breysse, E. D. Kovetz, P. S. Behroozi, L. Dai, and M. Kamionkowski, Insights from probability distribution functions of intensity maps, Mon. Not. R. Astron. Soc. 467, 2996 (2017).

[69] N. Aghanim, Y. Akrami, M. Ashdown, J. Aumont, C. Baccigalupi et al. (Planck Collaboration), Planck 2018 results: VI. Cosmological parameters, Astron. Astrophys. 641, A6 (2020).

[70] In the Fisher-matrix analysis, the variation of $\sum m_{\nu}$ and the change of the neutrino hierarchy are included in our fiducial model: we only consider deviations due to the neutrino decay and not to the varying neutrino masses.

[71] R. A. Fisher, The fiducial argument in statistical inference, Ann. Eugen. 6, 391 (1935).

[72] G. Jungman, M. Kamionkowski, A. Kosowsky, and D. N. Spergel, Weighing the Universe with the Cosmic Microwave Background, Phys. Rev. Lett. 76, 1007 (1996).

[73] G. Jungman, M. Kamionkowski, A. Kosowsky, and D. N. Spergel, Cosmological parameter determination with microwave background maps, Phys. Rev. D 54, 1332 (1996).

[74] M. Tegmark, A. N. Taylor, and A. F. Heavens, KarhunenLoève eigenvalue problems in cosmology: How should we tackle large data sets?, Astrophys. J. 480, 22 (1997).

[75] I. Esteban, M. Gonzalez-Garcia, A. Hernandez-Cabezudo, M. Maltoni, and T. Schwetz, Global analysis of threeflavour neutrino oscillations: Synergies and tensions in the determination of $\theta_{23}, \delta_{C P}$, and the mass ordering, J. High Energy Phys. 01 (2019) 106.

[76] V. Brdar, A. Greljo, J. Kopp, and T. Opferkuch, The neutrino magnetic moment portal: Cosmology, astrophysics, and direct detection, J. Cosmol. Astropart. Phys. 01 (2021) 039.

[77] M. Agostini et al. (Borexino Collaboration), Limiting neutrino magnetic moments with Borexino Phase-II solar neutrino data, Phys. Rev. D 96, 091103 (2017).

[78] J. M. Frere, R. B. Nevzorov, and M. I. Vysotsky, Stimulated neutrino conversion and bounds on neutrino magnetic moments, Phys. Lett. B 394, 127 (1997).

[79] M. Archidiacono, S. Hannestad, and J. Lesgourgues, What will it take to measure individual neutrino mass states using cosmology?, J. Cosmol. Astropart. Phys. 09 (2020) 021.

[80] Here we consider the LIM power spectrum and VID constraints separately, but they could be combined if the covariance between them is available [81].

[81] H. T. Ihle, D. Chung, G. Stein, M. Alvarez, J. R. Bond, P. C. Breysse et al., Joint power spectrum and voxel intensity distribution forecast on the $\mathrm{CO}$ luminosity function with COMAP, Astrophys. J. 871, 75 (2019).

[82] A. Merle and M. Totzauer, keV sterile neutrino dark matter from singlet scalar decays: Basic concepts and subtle features, J. Cosmol. Astropart. Phys. 06 (2015) 011.

[83] M. Shirasaki, Searching for eV-mass axion-like particles with cross correlations between line intensity and weak lensing maps, arXiv:2102.00580. 